\title{
O GeoGebra na construção do Pensamento Estatístisco: Transnumeração
}

\author{
Celina Aparecida Almeida Pereira Abar ${ }^{1}$, José Ronaldo Alves Araújo
}

${ }^{1}$ Program of Postgraduate studies in Mathematics Education - Pontifical Catholic University of São Paulo (PUC/SP) - São Paulo, SP - BRASIL

${ }^{2}$ Program of Postgraduate studies in Mathematics Education - Pontifical Catholic University of São Paulo (PUC/SP) - São Paulo, SP - BRASIL

abarcaap@pucsp.br, jronaldoaaraujo@gmail.com

\begin{abstract}
This article presents a doctoral research in progress in the context of Mathematics Education and focuses on the processes of Transnumeration and the use of GeoGebra. The work is the result of questions from an academic master's degree research in Mathematics Education. In an approximation to the theme, we bring considerations about statistical thinking, a discussion about its development in a computational environment and the understanding about Transnumeration. In this research we intend to apply sequences of activities supported by GeoGebra and the methodology of didactic engineering. We conjectured the possibility of constructing statistical thinking and, consequently, in the Transnumeration processes mediated by GeoGebra.
\end{abstract}

Resumo: Este artigo apresenta uma investigação de doutorado em andamento no âmbito da Educação Matemática e tem como foco os processos da Transnumeração e a utilização do GeoGebra. O trabalho é resultante de questionamentos oriundos de pesquisa de mestrado acadêmico em Educação Matemática. Em uma aproximação ao tema, trazemos considerações acerca do pensamento estatístico, uma discussão sobre seu desenvolvimento em um ambiente computacional e o entendimento sobre Transnumeração. Nesta pesquisa temos a intenção de aplicar sequências de atividades com suporte do GeoGebra e a metodologia da Engenharia Didática. Conjecturamos na possibilidade da construção do pensamento estatístico e por consequência, nos processos de Transnumeração mediados pelo GeoGebra.

\section{Introdução}

Este trabalho é parte de estudo de uma tese de doutorado em construção, no âmbito da Educação Matemática, e tem como objetivo apresentar algumas considerações acerca da Transnumeração, um tipo de pensamento estatístico, na perspectiva da utilização de tecnologias digitais. A motivação dessa pesquisa é decorrente de resultados obtidos na investigação de mestrado na qual foi desenvolvido um estudo sobre as Medidas de Tendência Central (MTC), a partir de uma sequência de atividades com suporte no software GeoGebra.

Naquela sequência de atividades observamos que os alunos, para responder a questionamentos a respeito de propriedades das MTC, recorriam às representações dos dados no GeoGebra para extrair estas medidas. O estudo, então, evidenciou que essas representações que o GeoGebra apresenta sobre dados é um aspecto favorável ao estudo das MTC.

Esse aspecto favorável é o que permite conjecturar para a possibilidade de elaboração de um constructo que apresente elementos da utilização de uma tecnologia digital para a construção de um tipo de pensamento estatístico, a Transnumeração.

A respeito da conjectura que trazemos, temos como questão norteadora: que processos 
transnumerativos resultam da resolução de atividades desenvolvida no GeoGebra? Este problema está diretamente ligado aos resultados apresentados em Araújo (2018), que sugere sequências de atividades que objetivem analisar esses processos de Transnumeração, decorrentes das representações de dados que uma tecnologia digital pode apresentar.

Diante da questão norteadora temos o objetivo de, por meio de sequências de atividades, realizar um estudo das estruturas do pensamento estatístico e da relação dos sujeitos com a tecnologia digital na tentativa de identificar aspectos relacionados à Transnumeração, constituídos a partir da manipulação de dados com suporte da tecnologia digital.

Buscamos justificar esta investigação na medida que observamos a quantidade de informações que nos chegam a todos os momentos, das mais variadas formas de representação que, na sua maioria, sugerem algum domínio de alguma tecnologia que permita compreendêlas. Nessa direção, consideramos que é necessário buscar meios que favoreçam a compreensão dessas informações e, mais ainda, buscar por meio dessas tecnologias, formas estruturais de pensamentos que afetam a compreensão das informações.

\section{Uma aproximacao ao tema}

Aqui nos referimos à construção do pensamento estatístico considerando o ensaio proposto por Wiild e Pfannkuch (1999), que consideram a Transnumeração como um tipo de pensamento estatístico, e apresentam aspectos que favorecem a compreensão das estruturas que formam o pensamento.

Nesse processo de estudo, nos aproximamos dos estudos de Ben-Zvi e Friedlander (1997), que apresentam elementos acerca do pensamento estatístico no ambiente tecnológico. Os estudos desses autores salientam para a influência do ambiente tecnológico nos processos cognitivos.

Diante da necessidade de aprofundar a compreensão acerca do uso de tecnologias, bem como a sua influência, em um primeiro momento nos referenciamos nos estudos de Lèvy (1993) que abordam as tecnologias da inteligência e nos permite conjecturar sobre as formas de como uma tecnologia digital pode favorecer aos processos transnumerativos.

Ainda acerca do uso de tecnologias, nos aportamos à Kenski (2003), que faz um estudo das tecnologias e a relação do ser humano com ela e nos aponta elementos relacionados ao processo de inserção das tecnologias à educação.

Quanto à utilização das tecnologias na educação, nesse ponto considerando a área de estudo que a pesquisa está sendo desenvolvida, nos aportamos para os estudos de Abar (2011) uma vez que esta pesquisadora apresenta considerações, que apontam para as tecnologias na Educação Matemática e corroboram para a assimilação que temos em relação ao uso das tecnologias digitais para o ensino de estatística.

A respeito do uso tecnologias digitais, para o ensino de estatística, Fey (1989) à época, salientou que o acesso aos computadores permite que os alunos trabalhem com coleções interessantes e realistas de dados numéricos. O autor observava que a comunidade de educação estatística se mostrava ativa na exploração desta oportunidade.

Há redes de computadores que dão aos usuários acesso a conjuntos de dados interessantes para instrução, há muitos softwares úteis disponíveis para procedimentos básicos de análise de dados e projetos de desenvolvimento de currículo estão mostrando como mesclar esses recursos de processamento de informações em instrução em todos os níveis [...] Talvez porque a análise de dados e as estatísticas não sejam vistas como estruturas tradicionais baseadas em habilidades no currículo, o debate sobre os riscos potenciais do uso de computadores parece não ter sido aguçado como em outros tópicos. O apelo e a promessa de expansão nessa 
VIII Congresso Brasileiro de Informática na Educação (CBIE 2019)

Anais dos Workshops do VIII Congresso Brasileiro de Informática na Educação (WCBIE 2019)

área geral parecem imenso (FEY, 1989, p. 242. Tradução nossa).

Como podemos observar o autor indicava a existência de softwares com potencial para tratar dados estatisticamente. Além disso, ao salientar para o desenvolvimento curricular, desde então já se tinha em conta, o interesse de fomentar a utilização desses avanços tecnológicos para o ensino da estatística.

Quando o autor aponta para a imensidão na perspectiva de ensino de estatística, bem mais adiante à época de Fey (1989), Bortolossi (2016), se referindo a um software específico, o GeoGebra, salienta para a existência de potencialidades do GeoGebra no processo de construção de significados para os objetos de estudo.

Estas potencialidades se evidenciam ao observar que, o GeoGebra, por meio de suas janelas, permite múltiplas representações dos dados, que o autor considera como uma vantagem didática. Nesse sentido, é que conjecturamos que uma tecnologia digital possa favorecer aos processos de Transnumeração.

\title{
3. Discussão teórica
}

Em primeiro momento, a necessidade de domínio do objeto de estudo nos direciona para nossos aportes teóricos, Wiild e Pfannkuch $(1999$, p. 227) ao se referirem à Transnumeração: "é um processo dinâmico de mudança de representações para gerar compreensão". Para os autores, o cunho da palavra Transnumeração refere-se à ideia fundamental em uma abordagem estatística para o aprendizado, a de formar e alterar representações de dados de aspectos de um sistema para chegar a um melhor entendimento desse sistema. Em outras palavras os autores a definem como "transformações de numeracia feitas para facilitar a compreensão" (WILD; PFANNKUCH, 1999, p. 227).

\begin{abstract}
A transnumeração ocorre quando encontramos formas de obter dados (por meio de medição ou classificação) que captam elementos significativos do sistema real. Ele permeia toda a análise de dados estatísticos, ocorrendo toda vez que mudamos nossa maneira de ver os dados, na esperança de que isso nos transmita um novo significado (WILD; PFANNKUCH, 1999, p. 227. Tradução nossa).
\end{abstract}

Nesse sentido, pode-se pensar em uma variedade de modelos estatísticos. "E no final do processo, a Transnumeração acontece mais uma vez quando descobrimos representações de dados que ajudam a transmitir nossos novos entendimentos sobre o sistema real para os outros". (WILD; PFANNKUCH, 1999, p. 227) .

Quando pensamos em uma variedade de modelos estatisticos, pensamos em associar a algum software como suporte para estudá-los, no entanto, não nos parece claro a noção da tecnologia e ainda mais considerando a estruturacao de modelos estatisticos. Partimos dessa discussão para introduzir Lèvy (1993), que indica o modelo digital de modo a permitir uma percepção, sobretudo, histórica, epstemológica da noção de tecnologia.

\footnotetext{
O modelo digital do qual nos servimos para fazer simulações encontra-se muito mais próximos dos bastidores da atiividade intelectual do que a cena teórica.[...] O que não impede as simulações de também desempenharem um papel de comunicação ou de persuasão importante, em particular quando a evolução do modelo é visualizada através de imagens em uma tela (LÈVY, 1993, p.126).
}

Aspectos apontados por Lèvy (1993) nos direcionam para os estudos de Ben-Zvi e Friedlander (1997) que, ao analisarem o pensamento estatístico sob a perspectiva de um ambiente tecnológico, asseveram que a tecnologia oferece a oportunidade de criar um ambiente de aprendizado totalmente novo, no qual os computadores podem ser usados como ferramentas na solução de problemas.

[...] a criação de um ambiente tecnológico de aprendizagem deve ter um impacto 
VIII Congresso Brasileiro de Informática na Educação (CBIE 2019)

Anais dos Workshops do VIII Congresso Brasileiro de Informática na Educação (WCBIE 2019)

considerável no conteúdo do currículo estatístico, e deve ser acompanhada de uma ampliação de seu enfoque na ênfase na compreensão conceitual, representações múltiplas e suas conexões, modelagem matemática, resolução de problemas, e maior atenção para aplicações do mundo real (CONSELHO NACIONAL DE PROFESSORES DE MATEMÁTICA ${ }^{1}$, 1989, apud, BEN-ZVI; FRIEDLANDER 1997)

É a partir do ponto de vista trazido por Ben-Zvi e Friedlander (1997), que aspectos metodológicos dessa investigação são norteados, uma vez que as ponderações apresentadas favorecem para a inserção de sequências de atividades em sala de aula.

\section{Metodologia}

Como mencionado introdutoriamente, em nossos objetivos, a investigação prevê a elaboração, aplicação e análise de sequências de atividades. Nesse sentido, metodologicamente, recorreremos a pressupostos da Engenharia Didática, para o desenvolvimento do estudo. Cabe ressaltar que, neste estudo, as atividades se referem à Estatística na Educação Básica, (ex: mediana, desvio padrão, desvio médio).

Os participantes da pesquisa serão alunos de uma escola pública do estado de São Paulo. O laboratório de informática será o ambiente de aplicação das sequências de atividades, que a priori utilizará como suporte, o GeoGebra.

\section{O desenvolvimento do estudo}

Este estudo se encontra em fase inicial e pretende-se que se desenvolva inicialmente com um levantamento dos estudos que circundam esta temática e considerando que o objeto de estudo está imbricado na ideia do pensamento estatístico buscaremos, também, fazer um levantamento dos estudos que se aproximam desse objeto com o uso das tecnologias digitais no ensino de estatística.

Como início desta pesquisa, buscamos levantar estudos a partir de palavras-chave como pensamento estatístico, ensino de estatística, tecnologias digitais e estatística, tecnologias e estatística e Transnumeração, direcionando os locais de busca.

No âmbito nacional, decidimos iniciar o levantamento de teses e dissertações, disponibilizadas no Banco de Teses da Coordenação de Aperfeiçoamento de Pessoal de Nível Superior-CAPES, pelo fato de a plataforma disponibilizar acesso a estudos realizados em todo do Brasil, e permite mapeá-los de forma abrangente. No âmbito internacional, recorremos ao Google Acadêmico, identificando além de teses e dissertações, artigos científicos que podem contribuir para nosso aprofundamento acerca do tema.

Estamos nesta fase de levantamento dos estudos e, portanto, ainda não foi possível quantificá-los, nem tampouco categorizá-los. No entanto, para apontar que trabalhos próximos do nosso objeto de estudo são temas de interesse para outros pesquisadores, apresentamos uma análise inicial acerca de uma tese de doutorado que conseguimos acessar por meio desse levantamento.

O tema pensamento estatístico foi abordado na tese de doutorado defendida por Silva (2007). A pesquisadora faz um estudo com professores de matemática aceca do Pensamento estatístico e raciocínio sobre variação. O estudo teve como objetivo verificar o raciocínio sobre variação e variabilidade nas etapas do ciclo investigativo do pensamento estatístico.

\footnotetext{
${ }^{1}$ National Council of Teachers of Mathematics,
} 
VIII Congresso Brasileiro de Informática na Educação (CBIE 2019)

Anais dos Workshops do VIII Congresso Brasileiro de Informática na Educação (WCBIE 2019)

Em um primeiro momento Silva (2007) expõe os que lhe parece ser os elementos do pensamento estatístico como sendo o reconhecimento da variação em todo o processo, a necessidade de dados para medir a variação e o uso de métodos e ferramentas estatísticas para quantificar e entender a variação, permitindo uma tomada de decisão.

A autora, para fundamentar o conceito de variação, apresenta considerações sobre o letramento estatístico, raciocínio estatístico e pensamento estatístico de modo a se situar no envolvimento da variação e seu papel nestes processos.

Acerca da noção de letramento estatístico, Silva (2007) recorre a Gal (2002) que expõe duas competências inter-relacionadas, a saber:

- Competência das pessoas para interpretar e avaliar criticamente a informação estatística, os argumentos relacionados aos dados ou a fenômenos estocásticos, que podem se apresentar em qualquer contexto e, quando relevante.

- Competência das pessoas para discutir ou comunicar suas reações para tais informações estatísticas, tais como seus entendimentos do significado da informação, suas opiniões sobre as implicações desta informação ou suas considerações acerca da aceitação das conclusões fornecidas. (GAL, 2002, apud SILVA, 2007, p. 24)

Após discutir as competências que Gal (2002) identifica no letramento estatístico, Silva (2007) traz a concepção de Garfield e Gal (2002, p.1) que explicitam sobre o raciocínio estatístico, a maneira como a pessoas raciocinam com ideias estatísticas e como percebem a informação estatística.

Acerca do pensamento estatístico, Snee (1990) define pensamento estatístico como o processo de pensamento que reconhece a presença de variação em torno de tudo o que se faz. Silva (2007) toma como referência Wild e Pffankuch (1999) ampliando esta definição apresentando uma estrutura baseada em quatro dimensões:

1) O ciclo investigativo que foi adaptado do modelo PPDAC (problem, plan, data, analysis, conclusions) de Wild e Pfannkuch (1999), que objetiva resolver um problema real, geralmente com a intenção de mudar o sistema para melhorar alguma coisa;

2) Os tipos de pensamento, o pensamento geral, que se refere ao planejamento do ciclo investigativo, o pensamento fundamental, que se refere ao reconhecimento das necessidades de dados, a Transnumeração, a consideração da variação a partir da tomada de decisão em situações de incertezas, o uso de modelos estatísticos e a integração da estatística num contexto;

3) O ciclo interrogativo, que diz respeito aos questionamentos macro e micro que são delineados pelo pensador enquanto resolve o problema e;

4) As disposições que se referem ao compromisso do pensador com problema.

Dessas condições Silva (2007) entende o pensamento estatístico como as estratégias mentais utilizadas pelo indivíduo para tomar decisão em toda etapa de um ciclo investigativo. Observamos que, nessas estratégias mentais é que podemos identificar e analisar os processos de Transnumeração.

\section{Considerações}

Embora o estudo se encontre em uma fase inicial salientamos que é possível observarmos a possibilidades de investigar os processos transnumerativos com a utilização de uma 
tecnologia digital, quando nos debruçarmos no estudo realizado por Silva (2007) acerca do conceito de variação e que nos aproxima das ideias de pensamento estatístico, ocorrendo por meio de estratégias mentais. O estudo nos permite observar que, conforme propõe o modelo PPDAC, a abordagem de um problema segue um ciclo, no qual há processos de construção desse pensamento estatístico, por consequência a Transnumeração.

Daí uma primeira conjetura seria considerar as possíveis representações de dados que o GeoGebra pode promover de modo a auxiliar na resolução de um problema dado nesse ciclo investigativo.

\section{Referências}

Abar, C. A. A. P. (2011) "Educação Matemática na Era Digital”. Unión Revista Iberoamericana de Educación Matemática, n. 27, p. 13-28.

Araújo, J. R. A. (2018) “Atividades para o estudo das Medidas de Tendência Central: uma proposta com o apoio do GeoGebra". Dissertação (Mestrado em Educação Matemática) Programa de Estudos Pós-Graduados em Educação Matemática. Pontifícia Universidade Católica de São Paulo, São Paulo. 145f.

Bortolossi, H. J. (2016) "O Uso do Software gratuito GeoGebra no Ensino e na Aprendizagem de Estatística e Probabilidade”. VIDYA, v. 36, n. 2, p. 429-440, jul./dez. Santa Maria, 2016. ISSN 2176-4603

Ben-zvi, D. \& Friedlander, A. (1997) "Statistical thinking in a technological environment". In J. B. Garfield \& G. Burrill (Eds.), Research on the role of technology in teaching and learning of statistics. Voorburg, the Netherlands: International Statistical Institute.

Fey, J. T. (1989) "Technology and Mathematics Education: A Survey of Recent Developments and Important Problems". Educational Studies in Mathematics. Information Technology and Mathematics Education. Vol. 20, n. 3, p. 237-272, Aug.

Lévy, P. (1993) “Tecnologias da Inteligência: o futuro do pensamento na era da Informática”. Tradução de Carlos Irineu da Costa. 1'. ed. São Paulo: Editora 34, 208 p.

Kenski, V. M. (2003) “Tecnologias e ensino presencial e a distância”. Campinas, SP: Papirus.

Silva, C. B. (2007) "Pensamento estatístico e raciocínio sobre variação: um estudo com professores de Matemática". Tese (Doutorado em Educação Matemática). Pontifícia Universidade Católica de São Paulo, São Paulo. 354f.

Wild, C. J. \& Pfannkuch, M. "Statistical Thinking in Empirical Enquiry". International Statistical Review, 67: 223-248, 1999. 